\title{
Acute cocaine-induced hepatotoxicity with features of shock liver
}

\author{
Demilade Adedinsewo, Oluwatosin Ajao, Ofego Okpobrisi, Claudia Fotzeu, Marvin Crawford \\ Department of Internal Medicine, Morehouse School of Medicine; Grady Memorial Hospital, Atlanta, GA, United States
}

Correspondence: Demilade Adedinsewo, MD. Address: 80 Jesse Hill J r. Dr. SE, Atlanta, GA 30303. United States. E-mail: dadedinsewo@msm.edu

Received: May 3, 2015

Accepted: July 8, 2015

Online Published: July 27, 2015

DOI : $10.5430 /$ crcp.v2n4p36

URL: http://dx.doi.org/10.5430/crcp.v2n4p36

\section{Abstract}

Background: Cocaine-induced toxicity has been described to be associated with severe adverse clinical health outcomes, affecting multiple organs systems with very limited clinical recommendations or guidelines for management of some of its associated complications.

Case report: We present a case of middle-aged female with acute severe hepatotoxicity after ingestion of alcohol and non-intravenous cocaine. She had markedly elevated liver enzymes and lactate dehydrogenase suggestive of shock liver but with minimal overt systemic manifestations. Patient was managed conservatively with intravenous fluids and treatment of con-current co-morbidities and liver enzymes and lactate dehydrogenase declined rapidly over a 5-day hospital course.

Discussion: We hypothesize that cocaine may play a role in diminishing hepatic perfusion, mimicking an ischemic hepatitis type clinical presentation and suggest that similar management, based on volume resuscitation, will potentially be more helpful than harmful for patient's presenting with acute cocaine-induced liver injury.

\section{Key words}

Cocaine, Hepatitis, Hepatotoxicity, Shock liver

\section{I ntroduction}

Cocaine toxicity is a well-known acute medical condition shown to affect multiple organ systems including the heart, muscle, kidney, brain, GI tract and the liver ${ }^{[1]}$. Cocaine-induced liver toxicity has been studied extensively in mice ${ }^{[2,3]}$ and reported in a few clinical cases among humans ${ }^{[4-6]}$. It has been described as a spectrum of disease ranging from minimal elevation of liver enzymes to severe hepatic failure ${ }^{[7]}$ and death. Severe hepatic injury resulting in marked elevation of liver enzymes and often in association with rhabdomyolysis, has been shown to result in poor outcomes including refractory hypotension, hypoglycemia, seizures, liver failure, disseminated intravascular coagulopathy, acute renal failure and death ${ }^{[4,5,8]}$. However to our knowledge, severe hepatic injury from cocaine use, with features of shock liver, resulting in spontaneous resolution and minimal to no health consequences has not been previously reported. We present a case of cocaine-induced acute hepatic injury in a middle-aged female in a metro area hospital. 


\section{Case presentation}

A 56-year-old woman presented to the emergency room with complaints of a four-day history of nausea and vomiting. Vomitus consisted of recently ingested food and was associated with non-bloody, loose stools occurring 4-5 times per day. She denied fever, recent travel, or recent contact with sick persons. She had consumed alcohol (4 cans of beer) and had used non-intravenous cocaine the day symptoms began. A review of systems revealed epigastric and lower abdominal pain, urinary frequency and urgency. Medical record review revealed urine drug screens were positive for cocaine in prior admissions. She had been diagnosed with chronic hepatitis C virus (HCV) infection five years earlier and at that time, her levels of serum transaminases, bilirubin, albumin, and international normalized ratio (INR) were normal. Her past medical history was significant for hypertension, anxiety, bipolar disorder and poly-substance abuse.

Physical examination revealed an anxious, restless and tremulous, overweight female. She was acutely ill looking, tachycardic with a heart rate of 106, and blood pressure of 135/96. She otherwise, had no significant abdominal exam findings. Laboratory results revealed markedly elevated aspartate aminotransferase (AST): 11,036 U/L; alanine aminotransferase (ALT): 3,796 U/L; lactate dehydrogenase: 5,751 U/L and mildly elevated gamma glutamyltransferase (194 U/L). The remainder of her metabolic profile, creatine kinase, complete blood cell count and cardiac troponin-I were essentially normal.

Urine toxicology screen on this admission was positive only for cocaine. Qualitative urinalysis revealed brown, cloudy urine, with a $\mathrm{pH}$ of $6.0,3+$ leucocyte esterase and negative for nitrite. Coagulation profile showed an INR of 1.4, a prothrombin time of 16.5 seconds and partial thromboplastin time of 26.7 seconds. Blood culture tests were negative but urine culture test results grew Proteus Mirabilis. Viral hepatitis serology revealed positive hepatitis A virus antibody (IgG), hepatitis B virus surface antibody (IgG), hepatitis B core antibody (IgG positive, IgM negative), and HCV antibody (IgG). She tested negative to hepatitis E antigen and antibody. An anti-nuclear antibody screen was negative. Serum ethanol, acetaminophen, valproic acid, ceruloplasmin and haptoglobin levels were within normal limits.

An electrocardiogram showed sinus tachycardia and abdominal ultrasonography showed minimal gallbladder sludge. Chest x-ray, abdominal x-ray and CT imaging of the abdomen showed no significant abnormalities. A needle liver biopsy showed chronic hepatitis B and C, grade III portal/periportal activity, grade II lobular activity and stage II fibrosis. The iron stain was negative with no evidence of granulomatous inflammation or neoplasia.

Based on the results of the work up discussed above, we were able to exclude hypovolemic hepatic ischemia (given our patient was hemodynamically stable on presentation), other drug induced liver toxicity (based on normal serum levels of ethanol, acetaminophen, valproic acid and urine drug screen negative for benzodiazepines), acute viral hepatitis (given markedly high transaminase levels $>50$ times the upper limit of normal, negative Hepatitis E and HBV core antibody IgM and the presence chronic HCV infection, diagnosed 5 years prior), Wilson's disease (based on normal serum ceruloplasmin levels and negative liver biopsy), hemochromatosis (based on negative iron stain on liver biopsy), autoimmune hepatitis (based on a negative ANA screen) and malignancy/metastatic disease (based on negative imaging tests and liver biopsy).

We also excluded other consequences of cocaine toxicity including rhabdomyolysis (given normal CPK levels), DIC (given normal blood count and coagulation profile), renal failure (given normal renal function on metabolic profile) and acute coronary syndrome (given no chest pain, negative troponin and EKG). As such, our assessment of pure cocaine induced hepatotoxicity was one of exclusion, which is an accepted standard for making a diagnosis of drug induced liver injury ${ }^{[9]}$.

Patient was managed conservatively with intravenous saline administration to ensure adequate hydration, avoidance of hepatotoxic medications, and treatment of her urinary tract infection with ciprofloxacin for 3 days. Her home medications 
included: venlafaxine and mirtazapine, fluoxetine and ziprasidone. Venlafaxine and mirtazapine were discontinued, as these were deemed excessive in relation to her medical conditions and inappropriate for management of her psychiatric illness. Her liver enzymes and serum LDH levels trended down rapidly over her hospital course (see Figure 1).

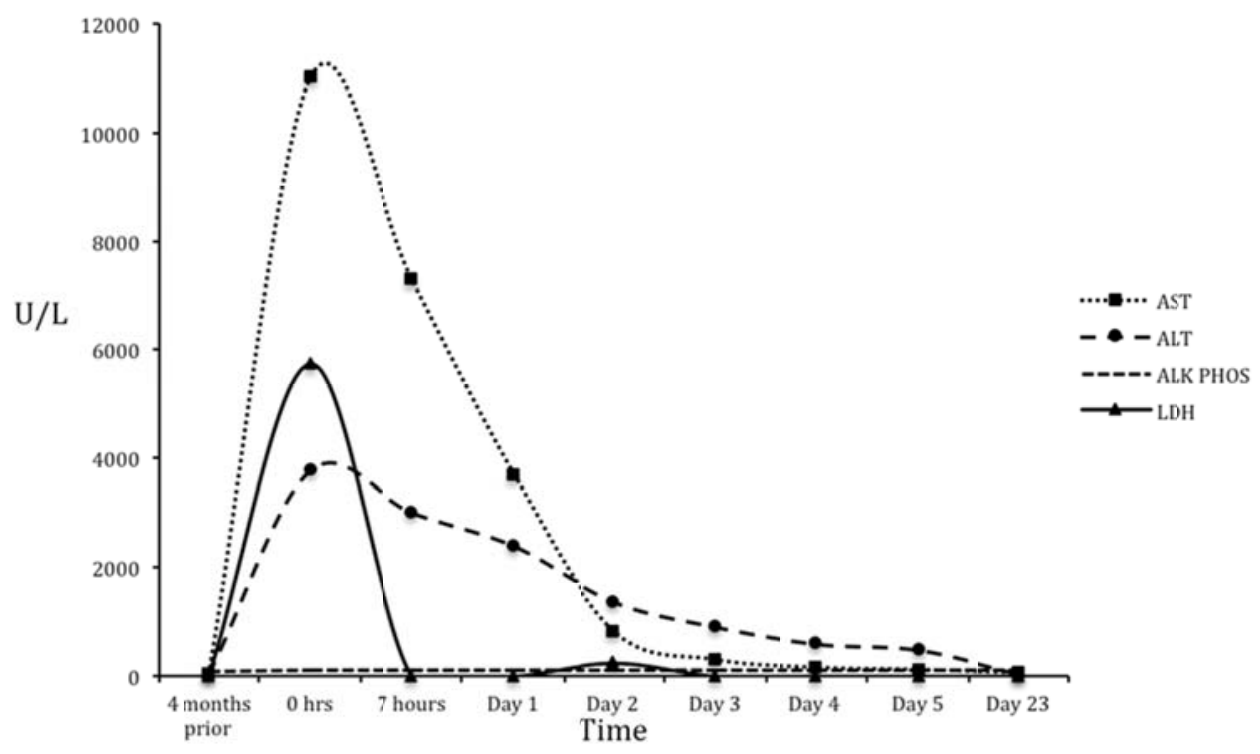

Figure 1. Trend in serum liver enzymes and lactate dehydrogenase (LDH) levels during hospital course, and at follow up post discharge

Note. Serum aspartate transaminase (AST), alanine transaminase (ALT), alkaline phosphatase (ALK PHOS) and lactate dehydrogenase (LDH) levels; at baseline (4 months prior to presentation), during hospital course, and at follow up, 18 days post discharge.

She was discharged from the hospital after five days, counseled to limit alcohol ingestion, avoid cocaine and medications not prescribed by her physician and encouraged to follow up with her primary care physician. Her liver enzymes, repeated by her primary care physician less than 3 weeks post discharge, were within normal limits; AST = 56 (normal range 10-42 $\mathrm{U} / \mathrm{L}$ ) and ALT = 38 (normal range 14-54 U/L) (see Figure 1).

\section{Discussion}

Our case report describes a patient with acute cocaine-induced toxic hepatitis without any other life threatening complications of cocaine toxicity, a rare case of pure hepatic cocaine toxicity with no overt systemic sequelae. Marked elevation of liver enzymes in this patient may have been potentiated by concurrent use of alcohol and cocaine. In experimental mice and human tissue studies, it has been shown that co-administration of alcohol synergistically enhances cocaine hepatotoxicity by increasing hepatic lipid peroxidation, free radical activity, hepatic metabolism of cocaine and reducing ethanol clearance, all proposed to be contributors to further liver damage ${ }^{[10-12]}$.

The hallmarks of cocaine-induced hepatitis are parenchymal damage with necrosis predominantly in zone 1 and 2 and accompanying microvesicular steatosis in areas of spared necrosis ${ }^{[1]}$. This was not demonstrated in our patient's liver biopsy; however, pathophysiologic and chemical findings were still attributable to cocaine-induced injury after excluding every other likely cause given the patient's history and clinical features. However, our patient's liver biopsy did show evidence of fibrosis, which is consistent with a similar case report demonstrating architectural distortion caused by fibrosis in a patient with cocaine-induced liver damage ${ }^{[13]}$. In addition, other reports of the liver findings seen with more severe cases of cocaine-induced hepatic toxicity were described post mortem ${ }^{[1,4]}$ and that may be a limitation to being able to observe these classical findings in our patient's liver biopsy which is limited to only the section of the liver biopsied. 
Our patient also had a history of poly-substance abuse and multiple unnecessary medication use, which may have contributed to acute elevation of liver enzymes and the clinical features seen. We cannot completely rule out the possibility of acute on chronic viral hepatitis in this patient as we were unable to obtain HAV IgM antibody values, however, it is unlikely that the severe marked elevation of liver enzymes to greater than 50 times the upper limit of normal as seen in this patient are attributable to acute viral hepatitis only. Also, the presence of marked elevation of LDH with rapid decline in transaminase levels is more in keeping with ischemic hepatitis ${ }^{[14]}$ likely to be acute drug- or toxin-induced as there was no evidence of hemodynamic instability in this patient on admission. Our patient also, did not manifest any evidence end-organ hypoperfusion, as expected with ischemic hepatitis ${ }^{[14]}$. The pathophysiology of ischemic hepatitis is still poorly understood $^{[15]}$, and we hypothesize that it may be possible, as seen with our patient, that cocaine may play a role in diminishing hepatic perfusion, mimicking an ischemic hepatitis type clinical presentation.

There is paucity of scientific evidence regarding the management of cocaine-induced hepatitis when compared to other sequelae of cocaine toxicity such as myocardial infraction, thrombotic microangiopathy, rhabdomyolysis and hypertensive crisis. Suggestions for management have mainly been conservative with prompt anticipation and identification of complications and appropriate management tailored to each patient ${ }^{[7]}$. An experimental study in mice suggests that induction of heme oxygenase may protect against cocaine-induced hepatic injury and may be key to developing an antidote in patients with acute cocaine ingestion to prevent hepatotoxicity ${ }^{[16]}$ or limit hepatic damage and its attending complications.

\section{References}

[1] Zimmerman H. Miscellaneous drugs and diagnostic chemicals. Hepatotoxicity the adverse effects of drugs and other chemicals. 1999; p. 709-42.

[2] Visalli T, Turkall R, Abdel-Rahman MS. Cocaine hepatotoxicity and its potentiation by lipopolysaccharide: treatment and gender effects. International journal of toxicology. 2004; 23(3): 163-70. Epub 2004/06/19. http://dx.doi.org/10.1080/10915810490471334

[3] Cascales M, Alvarez A, Gasco P, et al. Cocaine-induced liver injury in mice elicits specific changes in DNA ploidy and induces programmed death of hepatocytes. Hepatology (Baltimore, Md). 1994; 20(4 Pt 1): 992-1001. Epub 1994/10/01. PMid: 7927241. http://dx.doi.org/10.1002/hep.1840200431

[4] Kanel GC, Cassidy W, Shuster L, et al. Cocaine-induced liver cell injury: comparison of morphological features in man and in experimental models. Hepatology (Baltimore, Md). 1990; 11(4): 646-51. Epub 1990/04/01. PMid: 2328957. http://dx.doi.org/10.1002/hep.1840110418

[5] Hosseinnezhad A, Vijayakrishnan R, Farmer MJ. Acute renal failure, thrombocytopenia, and elevated liver enzymes after concurrent abuse of alcohol and cocaine. Clinics and practice. 2011; 1(2): e35. Epub 2011/05/16. http://dx.doi.org/10.4081/cp.2011.e35

[6] Balaguer F, Fernandez J, Lozano M, et al. Cocaine-induced acute hepatitis and thrombotic microangiopathy. Jama. 2005; 293(7): 797-8. Epub 2005/02/17. http://dx.doi.org/10.1001/jama.293.7.797

[7] Pateria P, de Boer B, MacQuillan G. Liver abnormalities in drug and substance abusers. Best practice \& research Clinical gastroenterology. 2013; 27(4): 577-96. Epub 2013/10/05. http://dx.doi.org/10.1016/j.bpg.2013.08.001

[8] Silva MO, Roth D, Reddy KR, et al. Hepatic dysfunction accompanying acute cocaine intoxication. Journal of hepatology. 1991; 12(3): 312-5. Epub 1991/05/01. http://dx.doi.org/10.1016/0168-8278(91)90832-V

[9] Teschke R, Frenzel C, Wolff A, et al. Drug induced liver injury: accuracy of diagnosis in published reports. Annals of hepatology. 2014; 13(2): 248-55. Epub 2014/02/21. PMid: 24552867.

[10] Odeleye OE, Watson RR, Eskelson CD, et al. Enhancement of cocaine-induced hepatotoxicity by ethanol. Drug and alcohol dependence. 1993; 31(3): 253-63. Epub 1993/02/01. http://dx.doi.org/10.1016/0376-8716(93)90008-E

[11] Pirozhkov SV, Eskelson CD, Watson RR. Chronic ethanol and cocaine-induced hepatotoxicity: effects of vitamin E supplementation. Alcoholism, clinical and experimental research. 1992; 16(5): 904-9. Epub 1992/10/01. PMid: 1443428. http://dx.doi.org/10.1111/j.1530-0277.1992.tb01891.x

[12] Ponsoda X, Jover R, Castell JV, et al. Potentiation of cocaine hepatotoxicity in human hepatocytes by ethanol. Toxicology in vitro: an international journal published in association with BIBRA. 1992; 6(2): 155-8. Epub 1992/03/01. 
[13] Payance A, Scotto B, Perarnau JM, et al. Severe chronic hepatitis secondary to prolonged use of ecstasy and cocaine. Clinics and research in hepatology and gastroenterology. 2013; 37(5): e109-13. Epub 2013/08/06. http://dx.doi.org/10.1016/j.clinre.2013.06.003

[14] Gitlin N, Serio KM. Ischemic hepatitis: widening horizons. The American journal of gastroenterology. 1992; 87(7): 831-6. Epub 1992/07/01. PMid: 1615936.

[15] Seeto RK, Fenn B, Rockey DC. Ischemic hepatitis: clinical presentation and pathogenesis. The American journal of medicine. 2000; 109(2): 109-13. Epub 2000/09/01. http://dx.doi.org/10.1016/S0002-9343(00)00461-7

[16] Ashino T, Sugiuchi J, Uehara J, et al. Auranofin protects against cocaine-induced hepatic injury through induction of heme oxygenase-1. The Journal of toxicological sciences. 2011; 36(5): 635-43. Epub 2011/10/20. PMid: 22008538. http://dx.doi.org/10.2131/jts.36.635 